\title{
UNDERWATER PHOTOGRAMMETRY IN VERY SHALLOW WATERS: MAIN CHALLENGES AND CAUSTICS EFFECT REMOVAL
}

\author{
P. Agrafiotis ${ }^{\text {a,c } *}$, D. Skarlatos ${ }^{\text {c }}$, T. Forbes ${ }^{b}$, C. Poullis ${ }^{\text {b }}$, M. Skamantzari ${ }^{c}$ and A. Georgopoulos ${ }^{c}$ \\ ${ }^{a}$ Cyprus University of Technology, Civil Engineering and Geomatics Dept., Lab of Photogrammetric Vision \\ 2-8 Saripolou str., 3036, Limassol, Cyprus \\ panagiotis.agrafioti@cut.ac.cy*, dimitrios.skarlatos@cut.ac.cy
${ }^{\mathrm{b}}$ Immersive and Creative Technologies Lab, Department of Computer Science and Software Engineering, Concordia University, Quebec H3G 1M8, Canada timforby@gmail.com, charalambos@poullis.org

\author{
${ }^{c}$ National Technical University of Athens, School of Rural and Surveying Engineering, Lab. of Photogrammetry \\ Zografou Campus, 9 Heroon Polytechniou str., 15780, Zografou, Athens, Greece \\ pagraf@central.ntua.gr, sk.margarita@hotmail.com,drag@central.ntua.gr
}

Commission II, WG II/9

KEY WORDS: Underwater 3D Reconstruction, SfM MVS, Caustics, CNN

\begin{abstract}
:
In this paper, main challenges of underwater photogrammetry in shallow waters are described and analysed. The very short camera to object distance in such cases, as well as buoyancy issues, wave effects and turbidity of the waters are challenges to be resolved. Additionally, the major challenge of all, caustics, is addressed by a new approach for caustics removal (Forbes et al., 2018) which is applied in order to investigate its performance in terms of SfM-MVS and 3D reconstruction results. In the proposed approach the complex problem of removing caustics effects is addressed by classifying and then removing them from the images. We propose and test a novel solution based on two small and easily trainable Convolutional Neural Networks (CNNs). Real ground truth for caustics is not easily available. We show how a small set of synthetic data can be used to train the network and later transfer the le arning to real data with robustness to intra-class variation. The proposed solution results in caustic-free images which can be further used for other tasks as may be needed.
\end{abstract}

\section{INTRODUCTION}

\subsection{Motivation}

Underwater 3D modelling and mapping techniques are based on various systems and methodologies but the most accurate of them are based on images as primary data. However, despite the relative low cost of such methods in relation to others, they suffer a major drawback; optical properties and illumination conditions of water severely affect image quality. Light is absorbed linearly to depth, resulting in a green-blue image due to strong absorption in red wavelength. Therefore, the red channel histogram has less information in comparison to green and blue. In addition, water absorbs light energy and scatters optical rays creating blurred images.

Even though the above phenomena affect RGB imagery in every depth, when it comes to shallow waters, caustics, the complex physical phenomena resulting from the projection of light rays being reflected or refracted by a curved surface, seems to be the main factor degrading image quality.

The implemented novel solution is based on two small and easily trainable CNNs (Convolutional Neural Networks). This approach demonstrates how a small set of synthetic data can be used to train the network and later transfer the knowledge to real data with robustness to intra-class variation. The implemented solution results in caustic-free images, which can be used for other applications. The above-mentioned approach is applied in a real world underwater site with depth varying from 0.5-1.5 meters. Imagery acquisition is characterized by intense caustics effect, which in many cases made matching on the initial imagery almost impossible. Moreover, in the following sections, the main challenges of underwater photogrammetry in shallow waters are described and analysed.

\subsection{Main challenges of underwater photogrammetry in shallow waters}

Underwater photogrammetry in shallow waters does not present some of the major problems of its counterpart in deep waters such as limited natural illumination, limited time etc., however, various other issues may affect the acquisition and processing stages.

\subsubsection{During the acquisition}

The definition of 'shallow' water is quite general. As a general rule, when the object is up to 10 metres in depth in clear water, water caustics by refraction effects may become a problem for all passive optical sensors. Unlike deep water photogrammetric approaches, where midday might be the best time for data capturing due to brighter illumination conditions, when it comes to shallow waters, the object to be surveyed needs strong artificial illumination, or images taken under overcast conditions, or with the sun low on horizon, in order to avoid lighting artefacts on the seabed. Besides lighting issues, during the acquisition phase, shallow water poses additional problems for diver based photogrammetric acquisition because of greater difficulty to control buoyancy (Bowens, 2011; Seinturier et al., 2004, Menna et al., 2013). To that direction, the waves may affect the stability of the diver and camera and change significantly its path. Similar effects may appear in deep water photogrammetric applications because of strong currents.

Usually in underwater photogrammetric tasks, a small camerato-object distance is selected to avoid absorption and achieve

\footnotetext{
* Corresponding author
} 
high resolution details and better accuracy. In shallow waters, where archaeological finds are usually ruins, the small camerato-object distance (less than $1 \mathrm{~m}$ ) is not always desirable or achievable. Really short camera-to-object distances lead to significant increase of acquired data, since the camera footprint on the seabed is reduced, the image scale is enlarged, thus resulting in higher processing time and larger storage requirement. In some extreme cases, of small depth there is not enough space for the diver and the camera. To overcome this, either cameras equipped with fisheye lenses are used, or two media photogrammetric approaches are adopted such as the one presented in Georgopoulos and Agrafiotis (2012). Other issues affecting the shallow water coastal area is the turbidity caused by the waves, floating objects etc.

\subsubsection{During processing}

The aforementioned problems during the acquisition phase, are also affecting processing of the collected data. Due to the small camera-to-object distance, a large amount of data has to be processed, increasing cost and time in real life applications. The above, together with caustics and illumination effects are affecting image matching algorithms and are the main cause for dissimilarities in the generated textures and orthoimages, if they are the final results. Regarding the caustics effect, which is the problem that is analysed in this paper, they throw off most of the image matching algorithms, leading to less accurate matches.

\section{IMPLEMENTED APPROACH FOR CAUSTICS REMOVAL}

\subsection{Related work}

For many years, the computer graphics research community has focused on the generation of caustics and as a result many techniques have been proposed which generate photorealistic results. At the same time only a few techniques have been proposed for the removal of caustics from images and video in the context of image enhancement. We provide a brief overview of the most relevant work to caustics removal. Trabes et al (Trabes et al, 2015) propose a technique which involves tuning a filter for sunlight-deflickering of dynamically changing underwater scenes. They employ a continuous parameter optimization inside a basic filter, which provides feedback for further improving the performance of the filter. Being an optimization the filter's performance is highly sensitive to suboptimal parameters and in particular, the segmentation parameter which is part of the objective function in the optimization. A different approach was proposed in (Gracias et al, 2008) where a mathematical solution was presented involving the calculation of the temporal median between images within a sequence. A strong assumption of this work, is the fact that feature matching [Harris corner detection variant in Gracias and Santos-Victor (2000)] is employed for the formation of the sequence which makes this approach very susceptible to the light variations in the images and in particular caustics effects.
The same authors later extend their work in (Shihavuddin et al, 2012) and propose an online sunflicker removal method which treats caustics as a dynamic texture. As reported in the paper this only works if the seabed or bottom surface is flat. Similar approaches have also been proposed for general cases of dehazing and descattering of images such as (Joshi et al, 2010), (He et al, 2011), (Fattal et al, 2008).

In (Schechner et al, 2004) the authors propose a method based on processing a number of consecutive frames. These frames are analyzed by a non-linear algorithm which preserves consistent image components while filtering out fluctuations. Their proposed method however does not take into account the camera motion which almost always leads to registration inaccuracies. Despite the innovative and complex aforementioned techniques, addressing caustic removal with procedural methods requires that strong assumptions are made on the many varying parameters involved e.g. scene rigidity, camera motion, etc.

\subsection{Convolutional Neural Networks Architecture}

The proposed solution in (Forbes et al, 2018) consists of two CNNs, SalienceNet and DeepCaustics and are described below.

SalienceNet: The input to SalienceNet is a rendered RGB image containing caustics of an underwater scene. The network operates on a batch of 32 images of size 400x400. Each pixel of the output image takes a value in the range of $[0,1]$, corresponding to the confidence of caustics occurring at that pixel. After extensive experimentation, we have concluded that the network architecture with the optimal performance consists of four hidden layers; the first two consisting of 3 and 5 convolution filters respectively, and the last two consisting of 5 and 1 de-convolution filters respectively. The filter sizes are $5 \times 5,3 \times 3,3 \times 3,5 \times 5$ in each layer respectively. This results in a total of $2 \times(5 \times 3 \times 3)+(4 \times 5 \times 5)$ weight parameters and $8+6$ bias parameters, for a total of 204 parameters to be learned. After each convolution/deconvolution in the network follows a ReLU activation unit. Initially, sigmoid activation units were used in the last layer to ensure that the final output is in the range $[0,1]$ however, our experiments have shown that ReLU units perform better [they still map the output in the range $[0,1]$ provided the input data falls within the manifold learned] and, in addition computing the gradients becomes more stable during backpropagation i.e. no 'squashing' leading to vanishing gradients. Adding more units and/or more layers has also been tested, but with no noticeable improvements. Larger filter sizes were also tested, but yielded blurry results. In order to get reasonable results with an initial layer consisting of larger filters, more layers with decreasing filter size were needed, but this required a reduction in the size of the images in the data set, due to memory constraints, and added no significant advantages. A diagram of the network's architecture, chosen based on all the experimental evaluations and considerations described above, is shown in Figure 1.
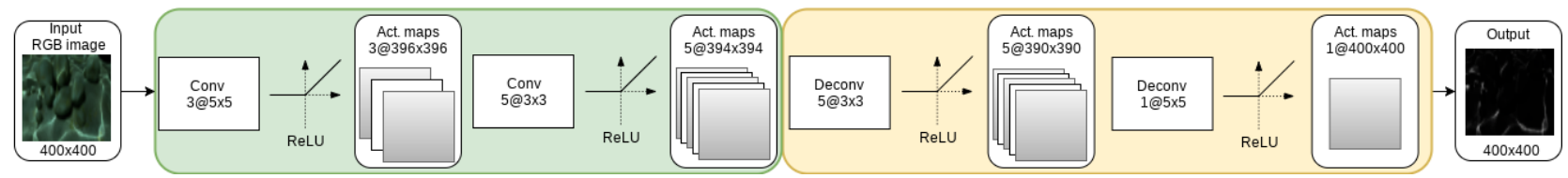

Figure 1. SalienceNet: a 4-layer CNN consisting of 2 convolutional layers followed by 2 deconvolutional layers. A ReLU activation unit follows each [de-]convolution operation. All [de-]convolution kernels have size $3 \times 3$. 
DeepCaustics: The input to DeepCaustics is the pair of an image containing caustics and the saliency map generated by SalienceNet. The two are first coupled together into a 4-channel RGBA format where the fourth channel contains the saliency value for the corresponding pixel. The ground truth used for training is a rendered caustic-free image corresponding to the synthetic input images. The network operates on a batch of 16 images of size $400 \times 400$. The output of the network is a causticfree RGB image corresponding to the input. After extensive experimentation, we have concluded that the network architecture with the optimal performance consists of six hidden layers; the first three consisting of 4,2 , and, 7 convolution filters respectively, the last three consisting of 7, 2, and 3 de- convolution filters respectively. The filter sizes are $3 \times 3,7 \times 7$, $3 \times 3,3 \times 3,7 \times 7,3 \times 3$ in each layer respectively. This results in a total of $(4 \times 3 \times 3)+2 \times(2 \times 7 \times 7)+2 \times(7 \times 3 \times 3)+(3 \times 3 \times 3)$ weight parameters and $(4+2 \times 2+2 \times 7+3)$ bias parameters, for a total of 410 parameters to be learned.

Similarly to SalienceNet, after each [de-]convolution in the network follows a ReLU activation unit. Figure 2 shows the architecture of the DeepCaustics network. The results of the proposed method are the original images without color transfer and histogram matching (Figure 3, middle column) and the caustic-free images generated by DeepCaustics on the color matched RGB images shown in Figure 3, right column.
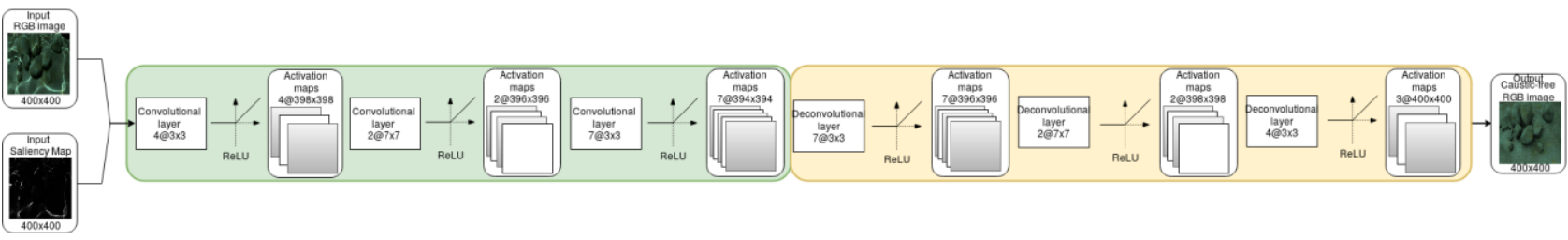

Figure 2. DeepCaustics: a 6-layer CNN consisting of 3 convolutional layers followed by 3 deconvolutional layers. A ReLU activation unit follows each [de-]convolution operation.

\subsection{Dataset used}

The imagery used presents a large variability in terms of colour, frequency and shape of the caustics. Data were acquired using two GoPro Hero 4 Black action cameras with image dimensions of $3000 \times 2250$ pixels, focal length of $2.77 \mu \mathrm{m}$ and pixel size of $1.55 \mu \mathrm{m}$. The dataset was captured in a near-shore underwater site at depths varying from 0.5 to 2 meters. No artificial light sources were used. Due to the wind, the turbulent surface of the water created dynamic sun flicker (caustics) on the seabed. Tests were performed using selected small datasets of different areas and depths over the test site. Five different datasets were used. One of them (the fifth in Figure 3, dataset 5), consists of video frames from the same camera, therefore image, i.e. frame dimensions are smaller: 1920 x 1080 pixels.

\section{APPLICATION AND RESULTS}

In Figure 3, results of the caustics correction algorithm are presented over a number of images. There, the original RGB images (uncorrected images), which contain caustics of varying characteristics, are presented in the first column, while the images which were processed using the network and then histogram matched to the colour transferred image (corrected $c t$ images) are presented in the middle column. Finally, in the right column, the caustic-free images which were processed using the network and then histogram matched to the original image are shown (corrected orig. images).

As it is obvious, in most of the datasets, the caustics have been strongly reduced and therefore further processing with SfMMVS techniques is possible.
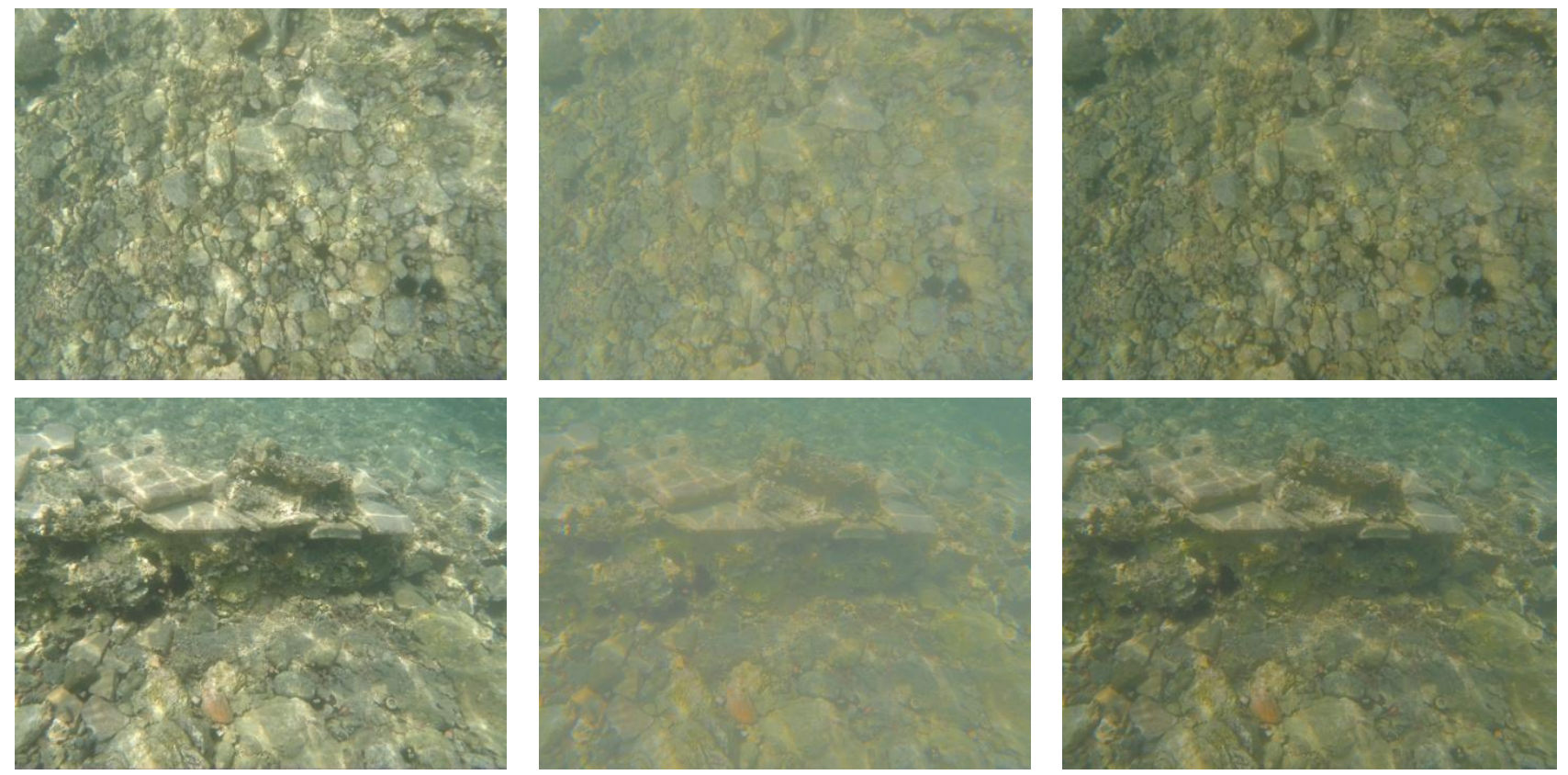

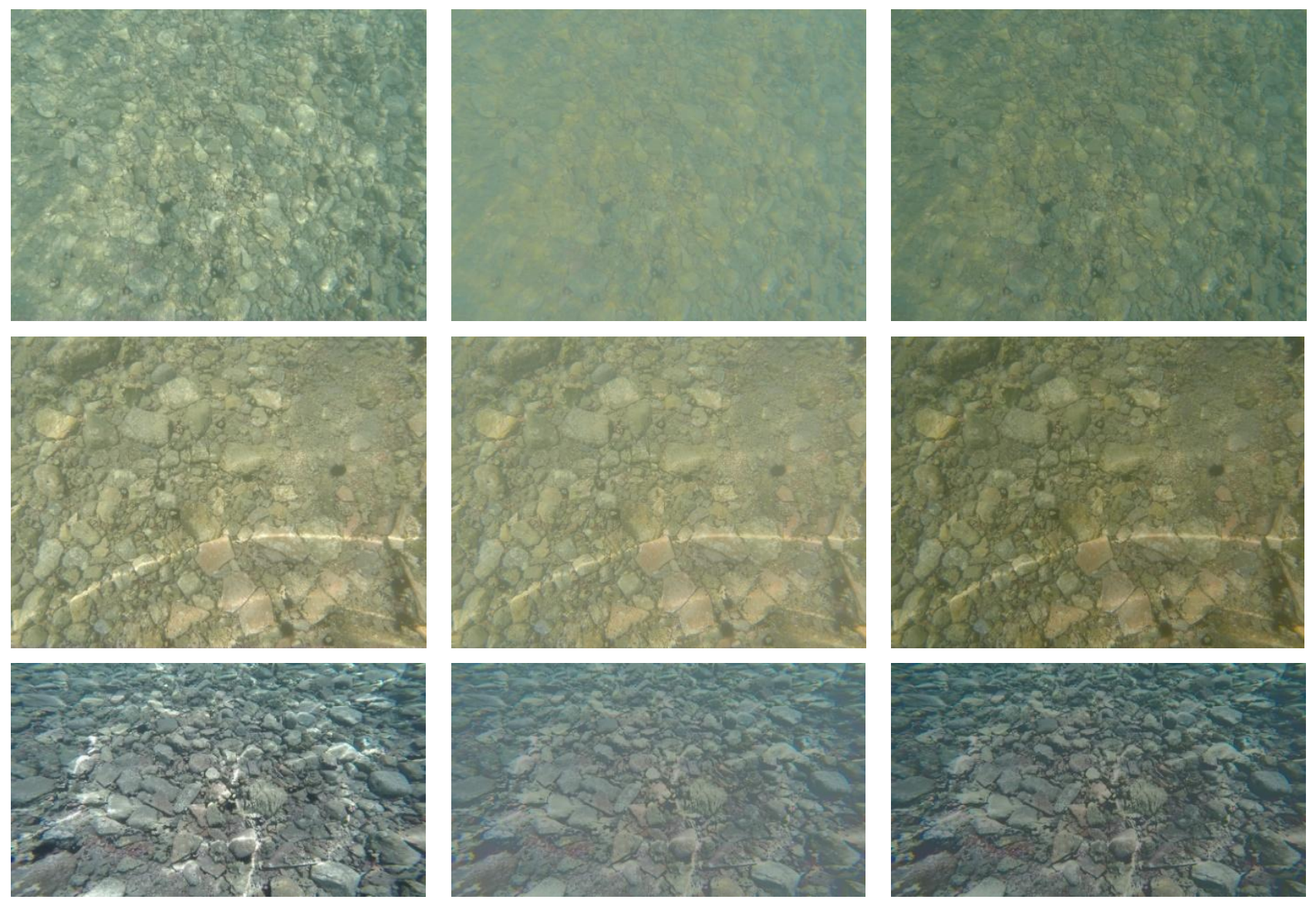

Figure 3. The five different datasets used: on the first row, an image from dataset 1, of the following rows, images from the respective datasets 2, 3, 4 and 5. Left column: The original RGB images, which contain caustics of varying characteristics. Middle column: The images which were processed using the network and then histogram matched to the colour transferred image. Right column: The caustic-free images which were processed using the network and then histogram matched to the original image.

\section{EVALUATION}

Apart from the visual evaluation of the results and their histograms comparison, the effectiveness of the applied method on caustics removal is evaluated by several tests for key point extraction and matching and 3D reconstruction through SfMMVS processes. 3D reconstruction was tested in Agisoft's Photoscan software while for key point matching, Agisoft's Photoscan software and other descriptors such as SIFT (Lowe, 1999) and SURF (Bay et al., 2006) were used. Main goal of this evaluation is to investigate how the caustics affect the matching process and the $3 \mathrm{D}$ reconstruction in order to extract valuable results about the proposed algorithm performance and caustics effect in general.

\subsection{Visual inspection/histograms}

Together with the visual inspection and evaluation of the caustic corrected imagery, the histograms of the five examples presented in Figure 3 are shown in Figure 4. There, the histogram of the green channel of the uncorrected image is plotted in magenta colour, the histogram of the green channel of the corrected ct image is plotted in yellow colour and finally, the histogram of the green channel of the corrected orig. image is plotted with green colour. In the red rectangular on each histogram, the peak around 250-255 representing the caustics effects is highlighted.
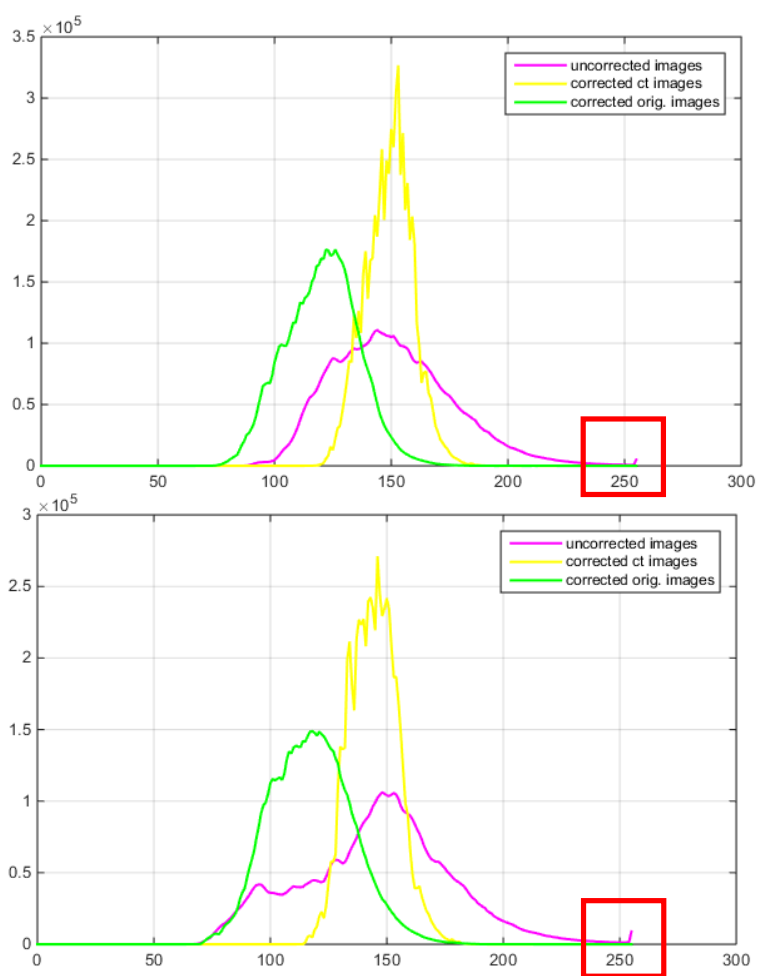

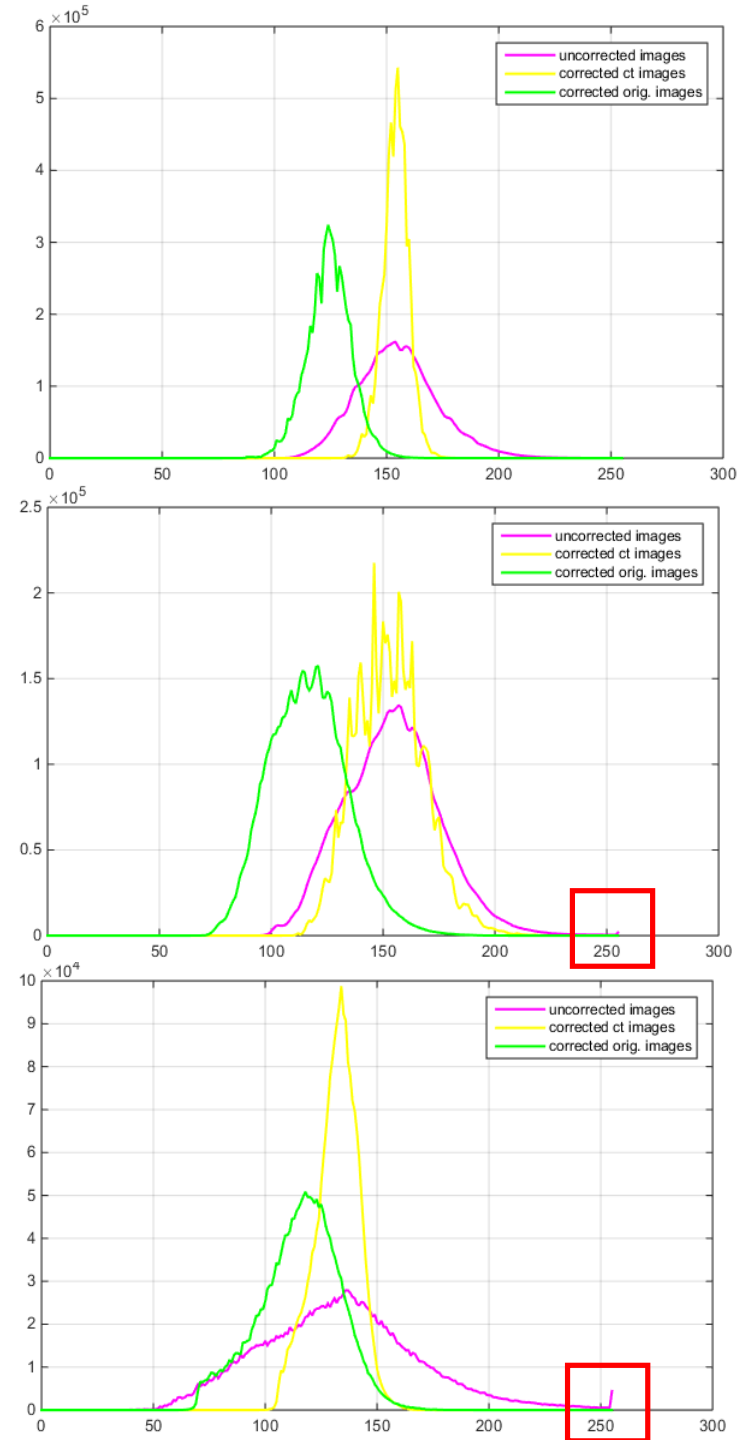

Figure 4. The respective histograms of the example images presented in Figure 3.

As it is observed, this peak does not exist in yellow and green histograms, which means that the caustics effect is strongly removed. Another fact observed is that in all of the cases, the final corrected image is darker than the initial one, since the majority of the brightness values are moved to the left.

\subsection{Key point matching}

The goal of this performed tests was to evaluate the effect of the caustics removal approach on SfM processing. For that, a commercial software performing SfM-MVS was used, the Agisoft's Photoscan as well as other key point descriptors such as SIFT (Lowe, 1999) and SURF (Bay et al., 2006). In the tests performed using the Agisoft's Photoscan, an image pair of the five different datasets was inserted and the alignment step was performed. Regarding the key point detection and matching, using the in-house implementations, the following procedure was followed, using exactly the same image pairs.

Feature Detection and Matching: Firstly, the key points were detected on the imagery. Scale Invariant Feature Transform (SIFT) (Lowe, 2004) extracts features invariant to image scale, rotation and translation and partially invariant to illumination changes. Speeded Up Robust Features (SURF) (Bay et al.,
2008) is based on the assessment of the Hessian matrix. SURF also combines both detection and description but it outperforms SIFT in terms of speed. Then, the similar kinds of features were identified in the scene. The detected features on the first image were then matched to the corresponding features on the second image and a mapping of these features between these two images was stored in a vector. This matching is based on nspace Euclidean distance, and performed both from left-to-right and right-to-left for redundancy.

Filtering of Matched points: Finally, the filtering of these matched points took place. In feature matching, several blunders might occur. The RANSAC algorithm is utilized to identify the inliers of the obtained point correspondences. The algorithm takes all the matched points as input, formulates a mathematical model that incorporates the majority of the points, and filters out the remaining points which are considered as outliers (Fischler and Bolles, 1987). To accomplish that, the fundamental matrix is computed and the measure for thresholding inliers points is the distance from the epipolar line. For this paper, the maximum distance from a point to an epipolar line in pixels, beyond which the point is considered an outlier was set at 3 pixels and the desirable level of confidence (probability) that the estimated matrix is correct to $99 \%$. At the end of this step, a set of matched points is found in the given scenes. Results of the matching process are given in Table 1. There, the total matched points and the valid ones -the output of the RANSAC filtering- are presented.
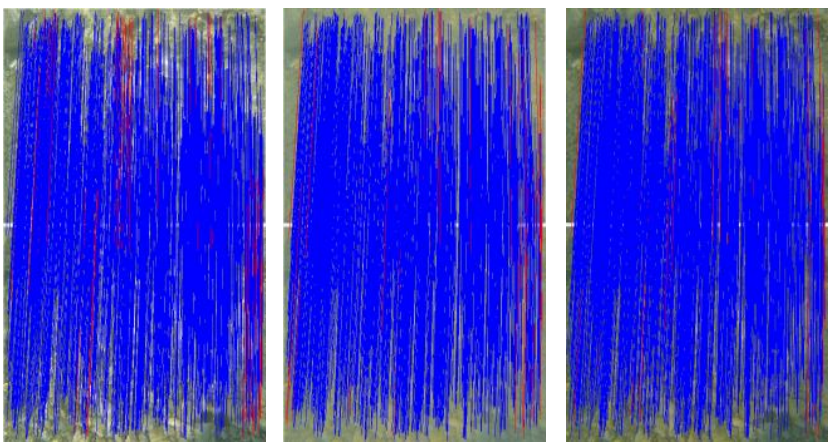

Figure 5. The matched points of an image pair from dataset 1 in Agisoft's Photoscan software.

As demonstrated in Figure 5, even the total matches are too many and it is not clear immediately, whether the corrected orig. image pair has more valid matches than the uncorrected one. This is also observed in most of the cases of Table 1 . Regarding SIFT and SURF results, two image pairs with their matches are presented in Figure 6 and Figure 7. In both figures, the left column contains SIFT results while the right column, SURF results. The first row contains the uncorrected image pairs, the second one the corrected ct image pairs and the third one the corrected orig. image pairs of the caustics free images. During the performed tests, it was decided not to evaluate the number of the total and valid matches only, but also evaluate the geometry of the matches, since some valid results of the RANSAC filtering are still matching the wrong points. Taking into account the above, one can observe in Figures 6 and 7 that the image pairs of the uncorrected imagery have a lot of intersecting matches, a phenomenon that is eliminated in the image pairs of the caustics free imagery (corrected ct image and corrected orig. image). This happens to all the tested datasets, however, for matter of space in this paper only the results of the dataset 1 and dataset 3 are presented. However, the matching results of all the datasets are presented in Table 1. 

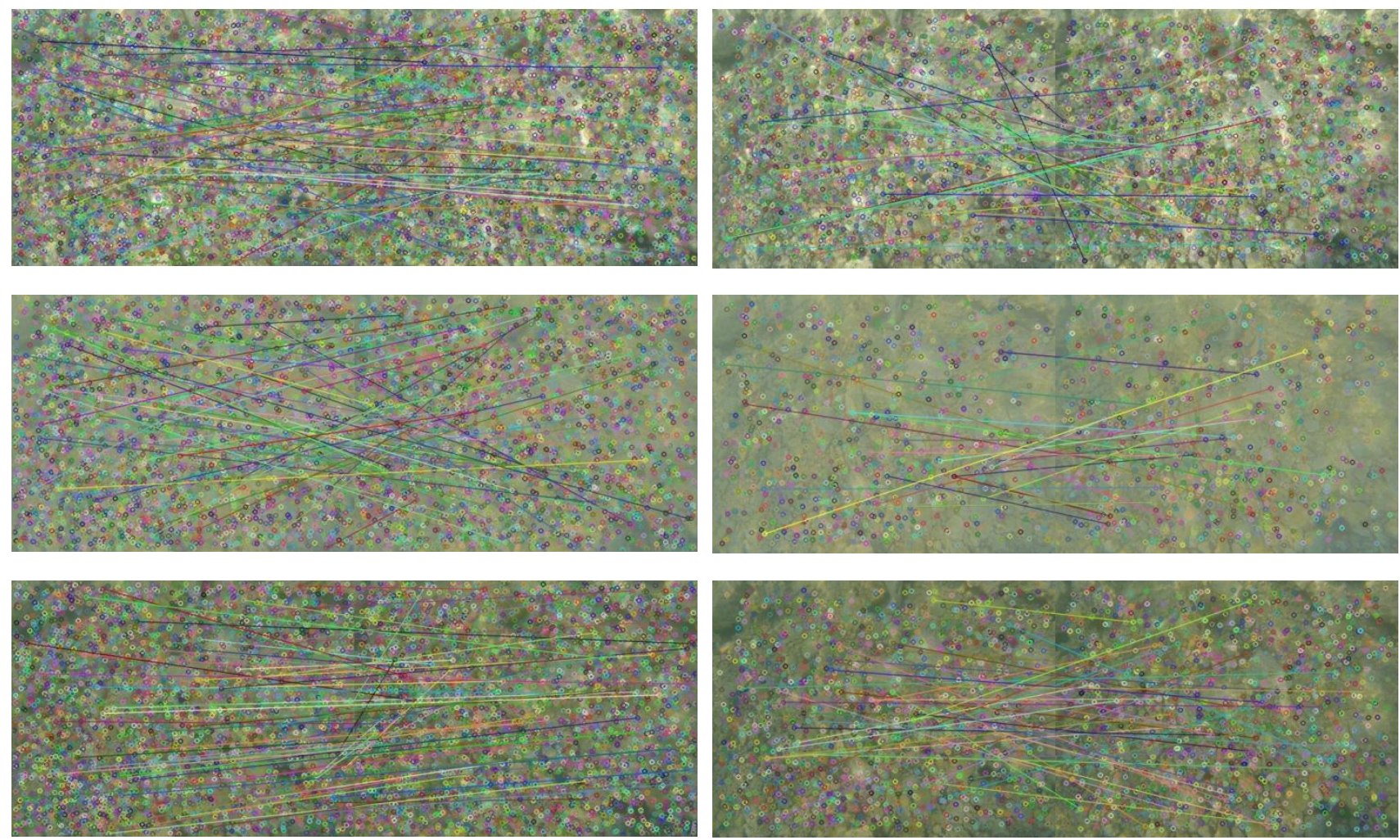

Figure 6. SIFT (left) and SURF (right) matched points on the original uncorrected imagery (first row), the corrected ct imagery (second row) and the corrected orig. imagery (third row) of the dataset 1.
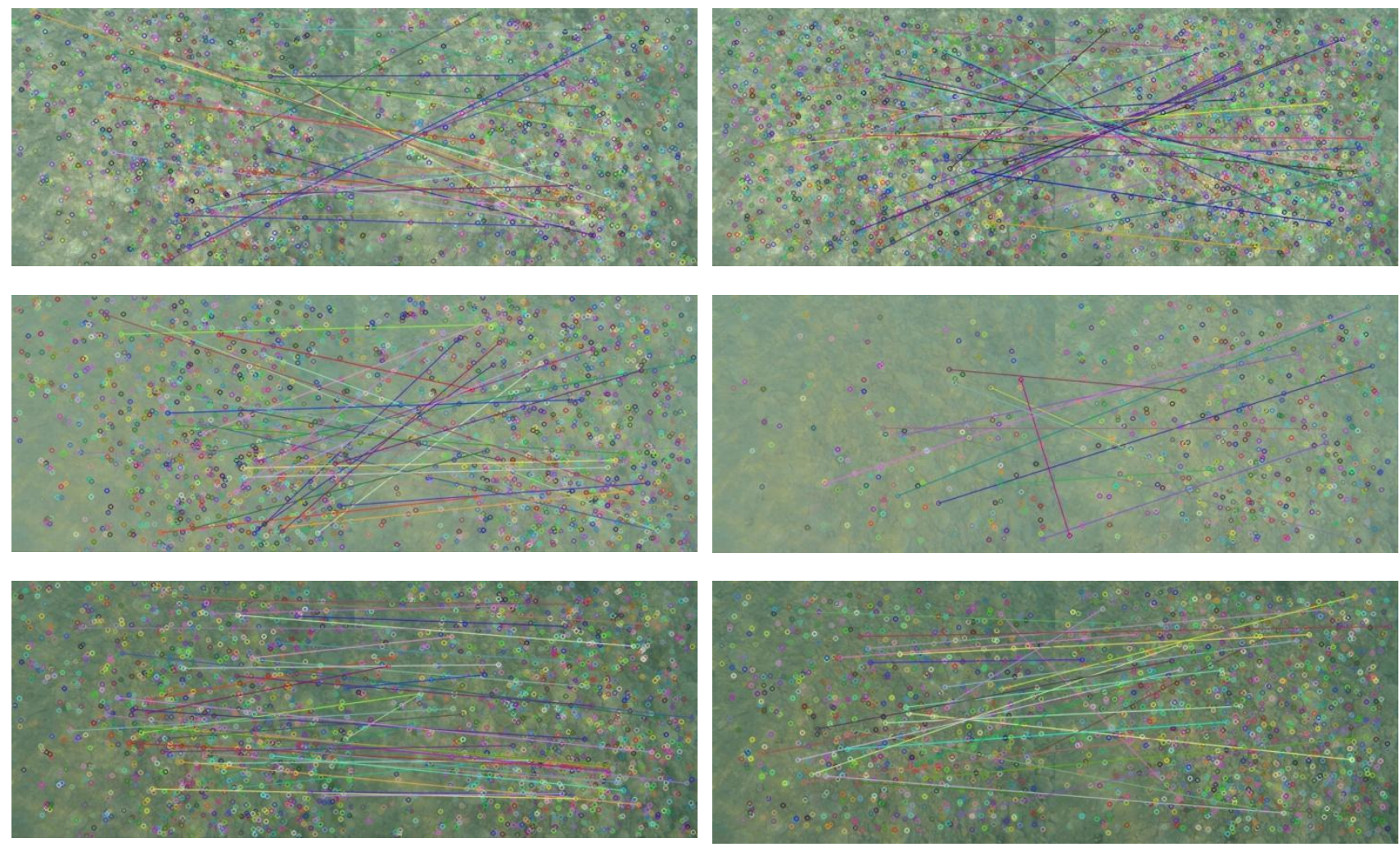

Figure 7. SIFT (left) and SURF (right) matched points on the original uncorrected imagery (first row), the corrected ct imagery (second row) and the corrected orig. imagery (third row) of the dataset 3. 


\begin{tabular}{lccccccc}
\hline & & \multicolumn{2}{c}{ Photoscan } & \multicolumn{2}{c}{ SIFT } & & \multicolumn{2}{c}{ SURF } \\
& & Total & Valid & Total & Valid & Total & Valid \\
\hline \multirow{3}{*}{ Dataset 1 } & Uncorrected images & 1345 & 1303 & 4041 & $\mathbf{6 6}$ & 1923 & 40 \\
& Corrected ct images & 1410 & 1379 & 3508 & 57 & 866 & 26 \\
& Corrected orig. images & 1423 & $\mathbf{1 3 9 9}$ & 3951 & $\mathbf{6 6}$ & 1505 & $\mathbf{4 5}$ \\
\hline \multirow{3}{*}{ Dataset 2 } & Uncorrected images & 741 & $\mathbf{7 3 6}$ & 3266 & 57 & 1695 & 41 \\
& Corrected ct images & 599 & 587 & 2268 & 41 & 471 & 21 \\
& Corrected orig. images & 597 & 588 & 3136 & 51 & 1157 & 28 \\
\hline \multirow{3}{*}{ Dataset 3 } & Uncorrected images & 1164 & 1120 & 4129 & 61 & 1611 & 39 \\
& Corrected ct images & 1206 & 1168 & 2352 & 44 & 164 & 14 \\
& Corrected orig. images & 1244 & $\mathbf{1 2 1 4}$ & 3676 & $\mathbf{6 2}$ & 810 & 28 \\
\hline \multirow{3}{*}{ Dataset 4 } & Uncorrected images & 1498 & 1428 & 3691 & 57 & 1741 & $\mathbf{3 6}$ \\
& Corrected ct images & 1476 & 1301 & 3606 & $\mathbf{6 0}$ & 1394 & 34 \\
& Corrected orig. images & 1473 & $\mathbf{1 4 3 7}$ & 3567 & $\mathbf{6 0}$ & 1533 & $\mathbf{3 6}$ \\
\hline \multirow{2}{*}{ Dataset 5 5} & Uncorrected images & 432 & $\mathbf{4 1 2}$ & 1067 & 37 & 645 & $\mathbf{3 1}$ \\
& Corrected ct images & 277 & 271 & 1059 & 39 & 245 & 22 \\
& Corrected orig. images & 306 & 296 & 1141 & $\mathbf{4 3}$ & 544 & $\mathbf{3 1}$ \\
\hline
\end{tabular}

Table 1 . The results of the matching process for all the datasets

There, in the $66 \%$ of the cases, the corrected orig. images are having more matches than the uncorrected images. However, even in the cases where the original imagery has more matches, these matches are wrong, compared with the respective ones on the caustics free images. The above results, suggest that the caustics effect, indeed affects the matching process in most of the cases. In the SIFT and SURF tests, results suggest that the caustic free images will facilitate a better image matching performance and thus better and more accurate 3D reconstruction.

\subsection{D reconstruction}

SfM-MVS Processing: Subsequently, all datasets were processed using SfM MVS with Agisoft's Photoscan commercial software. To this end, 5 different three-dimensional (3D) projects were created for each dataset and image dataset. At each site/project, 3 different blocks were created: (i) one with the uncorrected imagery, (ii) one with the corrected ct imagery and (iii) one with the corrected orig. imagery. All three channels of the images were used for these processes. For the created projects of each test site, the alignment parameters of the original (uncorrected) dataset were adopted to all other datasets. This ensured that the alignment parameters will not affect the $3 \mathrm{D}$ reconstruction and only the effect of the caustics on this specific process will be evaluated. Subsequently, 3D dense point clouds of medium quality and density were created for each data set. No filtering during this process was performed in order to get the total number of dense point clouds, as well as the noise. It should be noted that medium quality dense point cloud means that the initial images' resolution was reduced by a factor of 4 ( 2 times for each side), in order to be processed by the SfM-MVS software. The resulting point clouds were evaluated in terms of total number of points and roughness, a metric that indicates also the noise on the point cloud.

Total number of points: Here all the 3D points of the cloud were measured in order to get the total number, including any outliers and noise (Girardeau-Montaut, 2018).

Roughness: For each point, the roughness value is equal to the distance between this point and the best fitting plane computed based on its nearest neighbours (Girardeau-Montaut, 2018).
The performed experiments, suggest that the caustics removal and hence the processing of the imagery do not affect the generated point cloud in a negative way but they rather improve point cloud quality by slightly reducing its roughness and slightly increases the generated points by $2-3 \%$, as it is also demonstrated in Figure 8 and Figure 9.

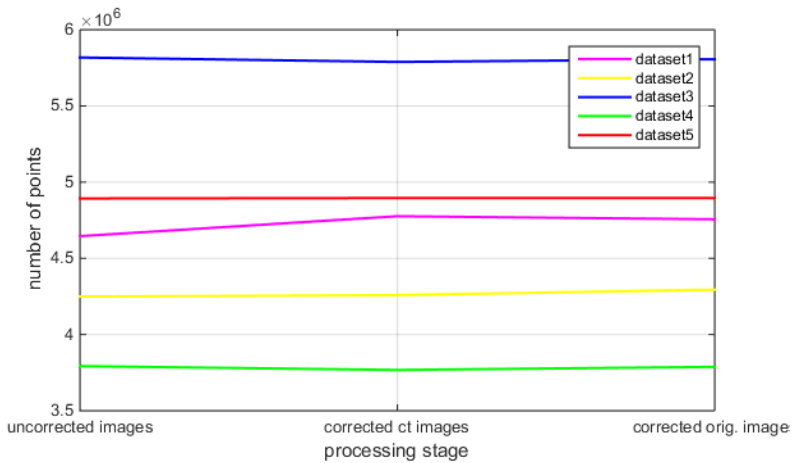

Figure 8 . The total number of points of each dataset for the 3 processing stages.

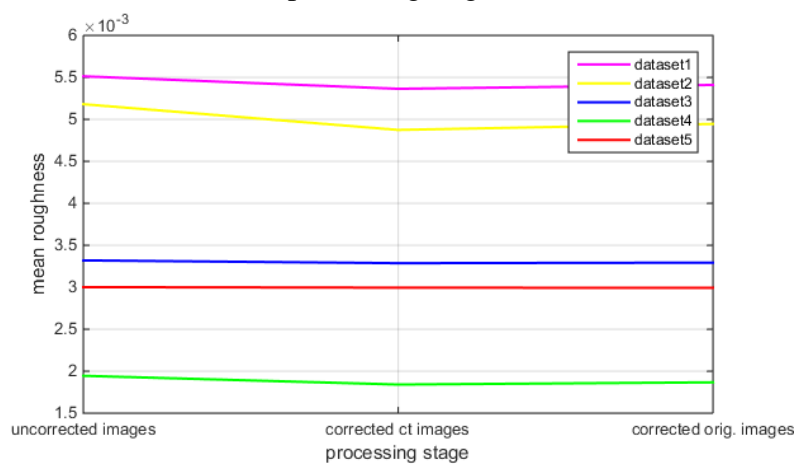

Figure 9. The mean roughness of the point cloud of each dataset for the 3 processing stages.

It is considered important that the unfiltered point clouds of the corrected imagery, having more points, have also less noise, as it is illustrated in Figure 10.

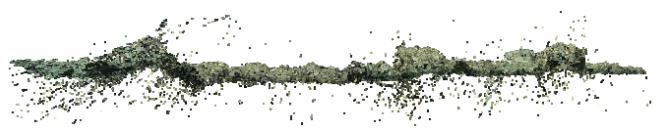

(a) 


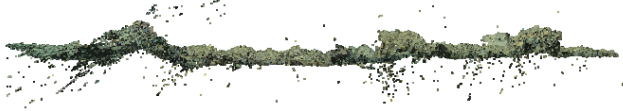

(b)

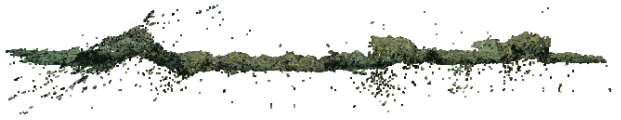

(c)

Figure 10. The unfiltered point clouds of the dataset 1. (a) the resulted point cloud using the uncorrected images, (b) the resulted point cloud using the corrected ct images and (c) the produced point cloud using the corrected orig. images.

\section{CONCLUDING REMARKS}

The implemented novel approach has been extensively tested on five real datasets of a shallow underwater site containing caustics. Test and evaluation indicate that caustics affect the key point detection and matching processes as well as 3D reconstruction and their removal is legitimate.

Results suggest that the implemented novel approach for caustics removal performs quite well in intense caustics, like the ones that are present in the dataset. As can be seen from the results, even with these relatively small networks and small synthetic training dataset we were able to transfer the learning to real world data quite effectively. As it is observed, in $66 \%$ of the cases, the total number of matches is increasing when the images are processed by the algorithm while in almost all of the cases the matches on the corrected orig. imagery are more reliable, even this is not the case for the corrected ct imagery. Most important is that the more successful the caustics removal is, the more valid matches are appearing in the stereo pair. Since the caustics have been successfully removed, further processing with structure-from-motion and multi-view stereo techniques becomes possible for a number of applications including underwater archaeology.

\section{ACKNOWLEDGEMENTS}

The contribution of P. Agrafiotis and D. Skarlatos is part of the i-MareCulture project (Advanced VR, iMmersive Serious Games and Augmented REality as Tools to Raise Awareness and Access to European Underwater CULTURal heritagE, Digital Heritage) that has received funding from the European Union's Horizon 2020 research and innovation programme under grant agreement No 727153. The design and development of the CNN networks is based upon work supported by the Natural Sciences and Engineering Research Council of Canada Grants No. N01670 (Discovery Grant). Authors would like to thank also the MSc student Alexandra Papadaki for her valuable contribution in key point matching and filtering.

\section{REFERENCES}

Bay, H., Tuytelaars, T., \& Van Gool, L., 2006. Surf: Speeded up robust features. Computer vision-ECCV 2006, 404-417.

Bowens, A. ed., 2011. Underwater archaeology: the NAS guide to principles and practice. John Wiley \& Sons

Fattal, R., 2008. Single image dehazing. ACM transactions on graphics (TOG), 27(3), 72.

Fischler, M. A., \& Bolles, R. C., 1987. Random sample consensus: a paradigm for model fitting with applications to image analysis and automated cartography. In Readings in computer vision (pp. 726-740).

Forbes, T., Goldsmith, M.,Mudur, S., Poullis, C., 2018, DeepCaustics: Classification and Removal of Caustics from Underwater Imagery, IEEE Journal of Oceanic Engineering (Under press)

Georgopoulos, A. and Agrafiotis, P,. "Documentation of a submerged monument using improved two media techniques," 2012. 18th International Conference on Virtual Systems and Multimedia, Milan, 2012, pp. 173-180. doi: 10.1109/VSMM.2012.6365922

Girardeau-Montaut, D.. 2018. Cloud compare - 3d point cloud and mesh processing software. Open Source Project.

Gracias, N., \& Santos-Victor, J., 2000. Underwater video mosaics as visual navigation maps. Computer Vision and Image Understanding, 79(1), 66-91.

Gracias, N., Negahdaripour, S., Neumann, L., Prados, R., \& Garcia, R., 2008. A motion compensated filtering approach to remove sunlight flicker in shallow water images. In OCEANS 2008 (pp. 1-7). IEEE.

He, K., Sun, J., \& Tang, X., 2011. Single image haze removal using dark channel prior. IEEE transactions on pattern analysis and machine intelligence, 33(12), 2341-2353.

Joshi, N., \& Cohen, M. F., 2010. Seeing Mt. Rainier: Lucky imaging for multi-image denoising, sharpening, and haze removal. In Computational Photography (ICCP), 2010 IEEE International Conference on (pp. 1-8). IEEE.

Lowe, D. G., 1999. Object recognition from local scaleinvariant features. In Computer vision, 1999. The proceedings of the seventh IEEE international conference on (Vol. 2, pp. 1150-1157). IEEE

Menna, F., Nocerino, E., Troisi, S., Remondino, F., 2013: A photogrammetric approach to survey floating and semisubmerged objects. Proc. of Videometrics, Range Imaging and Applications XII, SPIE Optical Metrology, Vol. 8791, doi: $10.1117 / 12.2020464$

Schechner, Y. Y., \& Karpel, N., 2004. Attenuating natural flicker patterns. In OCEANS'04. MTTS/IEEE TECHNOOCEAN'04 (Vol. 3, pp. 1262-1268). IEEE.

Seinturier, J., Drap, P., Durand, A., Vincent, N., Cibecchini, F., Papini, O. and Grussenmeyer, P., 2004. Orthophoto imaging and GIS for seabed visualization and underwater archaeology. In XXXII CAA (pp. 1-6).

Shihavuddin, A. S. M., Gracias, N., \& Garcia, R., 2012. Online Sunflicker Removal using Dynamic Texture Prediction. In VISAPP (1) (pp. 161-167).

Snavely, N., Seitz, S. M., \& Szeliski, R., 2008. Modeling the world from internet photo collections. International journal of computer vision, 80(2), 189-210.

Trabes, Emanuel, and Mario A. Jordan., 2015. "Self-tuning of a sunlight-deflickering filter for moving scenes underwater." Information Processing and Control (RPIC), 2015 XVI Workshop on. IEEE, 2015. 